Check for updates

Cite this: RSC Adv., 2017, 7, 48819

Received 13th June 2017

Accepted 3rd October 2017

DOI: 10.1039/c7ra06591d

rsc.li/rsc-advances

\section{Single Ni atom incorporated with pyridinic nitrogen graphene as an efficient catalyst for $\mathrm{CO}$ oxidation: first-principles investigation}

\author{
Mingguang Wang and Zhu Wang (DD)* \\ We have investigated the potential catalytic activity of a single $\mathrm{Ni}$ atom incorporated with pyridinic nitrogen \\ graphene (Ni-3N-G) in $\mathrm{CO}$ oxidation with first-principles calculations. It is found that the reaction pathway \\ would start with the Langmuir-Hinshelwood mechanism, and followed the progress of CO oxidation with \\ a remaining atomic $O$. The barriers for the formation and dissociation of a peroxo-type intermediate $O O C O$ \\ are 0.61 and $0.51 \mathrm{eV}$, respectively. The barrier that the remaining atomic $\mathrm{O}$ oxidizes $\mathrm{CO}$ is only $0.26 \mathrm{eV}$, \\ which makes $\mathrm{Ni}-3 \mathrm{~N}-\mathrm{G}$ regenerate. The results imply that the low-cost $\mathrm{Ni}-3 \mathrm{~N}-\mathrm{G}$ is a prospective catalyst \\ without noble metal for $\mathrm{CO}$ oxidation at room temperature.
}

\section{Introduction}

Earlier studies have found that some transition metal (TM) based catalysts can effectively catalyze $\mathrm{CO}$ oxidation, such as noble metal gold, ${ }^{1}$ platinum, ${ }^{2,3}$ palladium, ${ }^{4,5}$ rhodium, ${ }^{6,7}$ etc. However, these metal catalysts are expensive and rare, and then their mass production is restricted. Therefore, it is significant to lower the cost of these catalysts for $\mathrm{CO}$ oxidation reaction, or even reduce the energy barrier for this reaction.

Graphene, the unique two-dimension monolayer structure of $\mathrm{sp}^{2}$-hybridization carbon, has attracted the attention of researchers for its superior physical, chemical and mechanical properties in recent years. Meanwhile, the characteristic of the large specific surface area of graphene will be helpful to develop new catalysts. We expect that these catalysts can effectively remove toxic gases, such as CO, NO and so on. However, Z. M. Ao found that $\mathrm{CO}$ molecule is just has a very weak interaction with the intrinsic graphene. ${ }^{\mathbf{8}}$

Some experimental studies ${ }^{9-11}$ have shown that substitution doping is an effective way to improve the properties of graphene in various methods. Doping some metal $\left(\mathrm{Au},{ }^{12} \mathrm{Pt},{ }^{13} \mathrm{Cu},{ }^{14} \mathrm{Fe},{ }^{15}\right.$ etc.) or nonmetal (such as $\mathrm{N},{ }^{\mathbf{1 6}} \mathrm{P}^{\mathbf{1 7 , 1 8}} \mathrm{Si}^{19}$ ) atoms into graphene, theoretically, which could significantly enhance the activity of graphene and then show a good performance for CO oxidation reaction or oxygen reduction reaction (ORR). Interestingly, the $\mathrm{N}$-doped graphene (NG) demonstrates excellent electrocatalytic properties for ORR in alkaline fuel cells. ${ }^{20}$ Furthermore, some experimental $^{21,22}$ and theoretical ${ }^{23}$ studies have shown that TM modified NG is an effective cathode catalyst for ORR. In addition to this, some theoretical works proved that single transition

School of Physics and Technology, Hubei Nuclear Solid Physics Key Laboratory, Wuhan University, Wuhan, 430072, People's Republic of China. E-mail: wangz@whu.edu.cn; Tel: +8627 68752989-8025 metal atom incorporated with nitrogen-modified graphene ${ }^{24,25}$ were efficient catalysts for low temperature CO oxidation.

On the basis of the above evidences, we expect that $\mathrm{Ni}-\mathrm{N}$ codoped graphene may also show a comparable catalytic performance. In addition, $\mathrm{Ni}$ is readily available, which has a potential advantage to develop inexpensive and efficient catalyst. As we known, the study on the mechanism of CO oxidation by the Ni-N codoped graphene has been not reported yet. In present work, a new model of modified graphene without noble metal has been supposed, such as single Ni atom incorporated with pyridinic nitrogen graphene (Ni-3N-G). We simulate the possible reaction progress for $\mathrm{CO}$ oxidation on the surface of Ni-3N-G and investigate the mechanism of CO oxidation, to explore the possibility that Ni-3N-G acts as a catalyst for CO oxidation.

\section{Computational details and models}

Computational details: density functional theory (DFT) calculations coupled with the climbing image nudged elastic band method (CI-NEB) ${ }^{26-28}$ were used to study the mechanism for CO oxidation on Ni-3N-G. The calculations were operated with the Vienna $a b$ initio simulation package (VASP). ${ }^{29}$ Perdew-BurkeErnzerhof (PBE) exchange-correlation functional within the generalized gradient approximation (GGA) ${ }^{30}$ were applied in the calculations. Bader charge analysis ${ }^{31}$ was used to evaluate the atomic charges and the electron transfer in the reactions. Our calculation model is based on the pristine $4 \times 4$ hexagonal graphene supercell, which includes 32 atoms totally. Then the supercell was modified with three pyridinic nitrogen atoms and a nickel atom. In the geometric relaxation and CI-NEB calculations, the Brillouin zone (BZ) integration was performed with a $3 \times 3 \times 1$ Monkhorst-Pack $k$-point mesh. The wave functions were expanded by plane waves with an energy cutoff of $400 \mathrm{eV}$. 
The convergence criteria for electronic and ionic relaxation are $1 \times 10^{-5} \mathrm{eV}$ and $0.02 \mathrm{eV} \AA^{-1}$, respectively. For avoiding the interactions between the graphene sheet and its mirror images, the distance of them was set to $15 \AA$ A. The CI-NEB was employed to search the minimum energy paths (MEP).

The binding energy $\left(E_{\mathrm{b}}\right)$ of $\mathrm{Ni}$ atom onto NG can be calculated using the following equation:

$$
E_{\mathrm{b}}=E_{\mathrm{Ni}-\mathrm{NG}}-\left(E_{\mathrm{Ni}}+E_{\mathrm{NG}}\right)
$$

In eqn (1), $E_{\mathrm{Ni}-\mathrm{NG}}$ stands for the total energy of the $\mathrm{Ni}$ atom dispersed on NG, $E_{\mathrm{Ni}}$ and $E_{\mathrm{NG}}$ stand for the energy of free $\mathrm{Ni}$ atom and clean NG.

The adsorption energy between the adsorbate and substrate can be calculated using the following equation:

$$
E_{\text {ads }}=\left(E_{\text {substrate }}+E_{\text {adsorbate }}\right)-E_{\text {substrate/adsorbate }}
$$

In eqn (2), $E_{\text {substrate/adsorbate }}$ and $E_{\text {substrate }}$ represent the total energy of substrate with or without adsorbate, respectively. $E_{\text {adsorbate }}$ stands for the energy of free adsorbate (such as $\mathrm{CO}, \mathrm{O}_{2}$, $\mathrm{CO}_{2}$ and $\mathrm{O}$ ) in its ground state. According to this equation, a positive adsorption energy corresponds a stable adsorption structure.

\section{Results and discussion}

\subsection{Studies on the stability of $\mathrm{Ni}$ incorporated with pyridinic nitrogen graphene}

$\mathrm{Liu}^{24}$ investigated Pt-N codoped graphene by first-principles, which found that introducing pyridinic nitrogen can stabilize defect, and the dangling bonds could be completely vanished when the vacancy was surrounded by three pyridinic nitrogen atoms to generate a new structure (marked as pyri-3N-G). Furthermore, they found that the pyri-3N-G could stabilize the deposited Pt atom effectively and then shows a good catalytic performance for $\mathrm{CO}$ oxidation. The similar results about catalytic performance for $\mathrm{CO}$ oxidation were presented by Zhang. ${ }^{25}$

In order to investigate the stability of Ni-3N-G substrate, we first study the adsorption of single $\mathrm{Ni}$ atom on the pyri-3N-G. Four possible adsorption sites are supposed (as shown in Fig. 1a), i.e. the center of a carbon hexagon (C1-site), the center of a pyridine ring (C2-site), the center of carbon-carbon bond (B-site) and the vacancy which surrounded by 3 pyridinic $\mathrm{N}$ atoms (V-site). A nickel atom was successively placed at one site of the four supposed sites with a height of $2 \AA$ above the pyri-3N$\mathrm{G}$ plane. Then, we test the stability that single $\mathrm{Ni}$ atom is adsorbed at each site, respectively.

After full relaxation, all of the optimized geometric configurations are depicted in Fig. 1 and the related information is listed in Table 1. Our calculation results indicate that the single $\mathrm{Ni}$ atom could be adsorbed on each of four possible adsorption sites, respectively. When the single Ni atom adsorbed on the Vsite, it has the smallest $h$ value and the lowest $E_{\mathrm{b}}$ value in comparison to three other adsorption sites. Then, the V-site could be considered as the most favorable adsorption site for single $\mathrm{Ni}$ atom adsorbed on the pyri-3N-G. The $\mathrm{Ni}$ atom
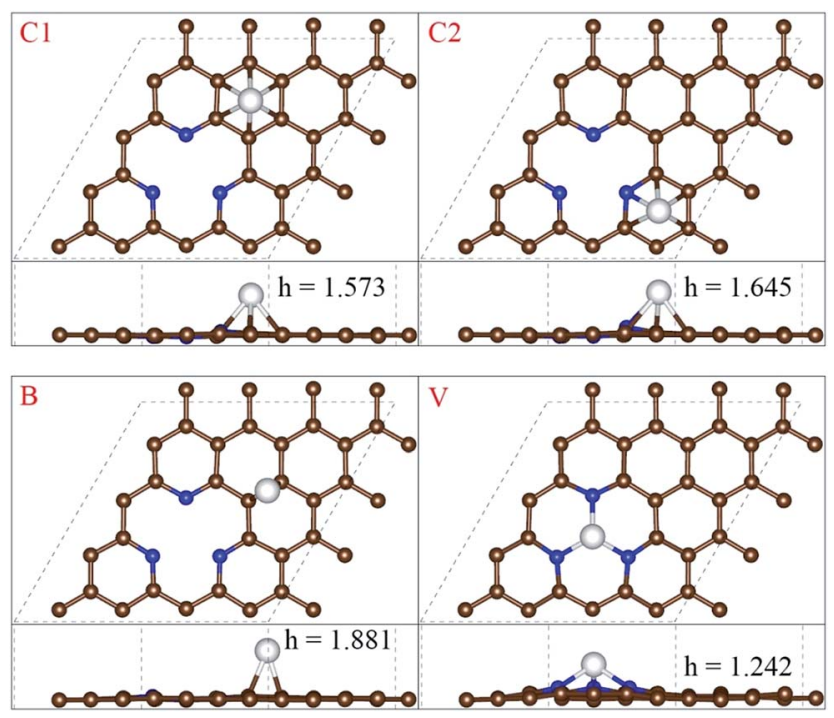

Fig. 1 The geometry configurations of four different adsorption sites for single $\mathrm{Ni}$ atom on the pyri-3N-G after relaxation. The brown, blue and white spheres represent the $\mathrm{C}, \mathrm{N}$ and $\mathrm{Ni}$ atoms, respectively.

Table 1 Binging energy $\left(E_{\mathrm{b}}\right)$ and adsorption height $(h)$ for single $\mathrm{Ni}$ atom adsorbed on each possible adsorption site $(C 1, C 2, B, V)$ after relaxation. Here $h$ represents the height of $\mathrm{Ni}$ atom above the pyri-3NG plane

\begin{tabular}{lll}
\hline & $E_{\mathrm{b}}$ & $h$ \\
\hline $\mathrm{C} 1$ & $-1.868 \mathrm{eV}$ & $1.573 \AA$ \\
$\mathrm{C} 2$ & $-1.754 \mathrm{eV}$ & $1.645 \AA$ \\
$\mathrm{B}$ & $-1.690 \mathrm{eV}$ & $1.881 \AA$ \\
$\mathrm{V}$ & $-4.451 \mathrm{eV}$ & $1.242 \AA$
\end{tabular}

adsorbed on the V-site with an average Ni-N bond length of 1.85 $\AA$, which is smaller by about $0.24 \AA$ than that of $\mathrm{Pt}-\mathrm{N}$ bond length, ${ }^{24}$ due to the smaller atomic radii of $\mathrm{Ni}$ than Pt. In addition, as shown in Fig. 2b, Bader charge analysis results show the pyri-3N-G substrate has gained about $0.13 e$ from $\mathrm{Ni}$ atom.

Considering the possibility of $\mathrm{Ni}$ atom escaping from the $\mathrm{V}$ site, we need to simulate the diffusion process of $\mathrm{Ni}$ atom to its adjacent other possible adsorption sites. The V-site was chosen as the initial state (IS), and the other three adsorption sites (such as C1, C2 and B-site in Fig. 2a) as the final state (FS). Here the CI-NEB calculations was used to search the MEP from IS to FS. The corresponding diffusion barriers are $2.811 \mathrm{eV}$ (from V to C1-site), $2.917 \mathrm{eV}$ (from V to C2-site) and $2.829 \mathrm{eV}$ (from V to Bsite), respectively. The higher diffusion energy barriers indicate that $\mathrm{Ni}$ atom at $\mathrm{V}$-site is difficult to escape, which further confirm the stability of Ni-3N-G.

\subsection{The adsorption of the reactants}

Due to the adsorption of the reactants is a prerequisite for $\mathrm{CO}$ oxidation, we first study the adsorption properties of various adsorbates $\left(\mathrm{O}_{2}, \mathrm{CO}, \mathrm{CO}_{2}\right.$ and $\left.\mathrm{O}\right)$ on the optimal structure of $\mathrm{Ni}-$ 


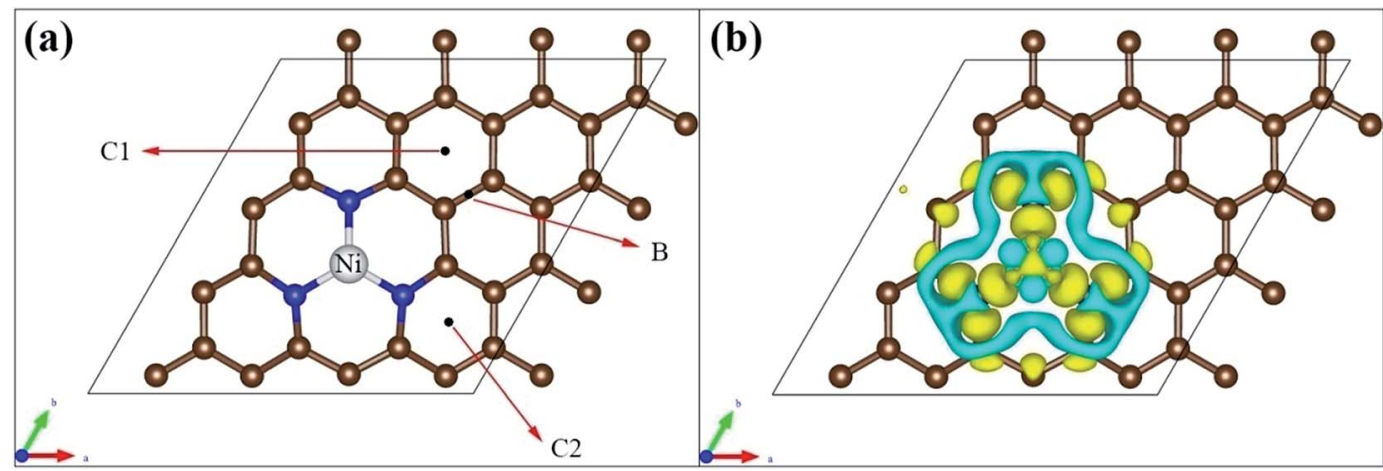

Fig. 2 The optimized geometric configuration of Ni-3N-G (a) and its charge density difference (b). The brown, blue and white spheres represent the $\mathrm{C}, \mathrm{N}$ and $\mathrm{Ni}$ atoms, respectively. The faint yellow and azure areas represent charge accumulation and depletion, respectively.

Table 2 Adsorption energy $\left(E_{\text {ads }}\right)$ of various adsorbates on $\mathrm{Ni}-3 \mathrm{~N}-\mathrm{G}$ and the number of electrons $(\Delta q)$ transferred from Ni-3N-G substrate to the adsorbate

\begin{tabular}{lll}
\hline Adsorbate & $E_{\text {ads }}(\mathrm{eV})$ & $\Delta q(\mathrm{e})$ \\
\hline $\mathrm{O}_{2}$ & -2.06 & 1.02 \\
$\mathrm{CO}$ & -2.04 & 0.26 \\
$\mathrm{CO}_{2}$ & -0.16 & 0.03 \\
$\mathrm{O}$ & -4.81 & 0.61 \\
$\mathrm{CO}+\mathrm{O}_{2}$ & -2.70 & 0.50
\end{tabular}

$3 \mathrm{~N}-\mathrm{G}$. In order to find out the most stable configuration for each adsorbate, we tested many adsorption sites. The adsorption energy at the most stable site and related information for each adsorbate are listed in Table 2.

For $\mathrm{O}_{2}$ adsorption, the most stable configuration (as shown in Fig. 3a) with $E_{\text {ads }}\left(\mathrm{O}_{2}\right)=-2.06 \mathrm{eV}$, the distance between the two $\mathrm{O}$ atoms of $\mathrm{O}_{2}$ is prolonged by $0.138 \AA$ (from 1.234 to $1.372 \AA)$. We find that a large amount of charge is gathered near $\mathrm{O}_{2}$ and 1.02e charge transferred from the substrate to $\mathrm{O}_{2}$.
For $\mathrm{CO}$ adsorption, the most stable configuration with $E_{\text {ads }}(\mathrm{CO})=-2.04 \mathrm{eV}$. The electrons accumulate on both $\mathrm{C}-\mathrm{Ni}$ bond and on $\mathrm{O}$ ion. Meanwhile, $\mathrm{CO}$ gains $0.26 e$ charge from the substrate and the distance of $\mathrm{C}-\mathrm{O}$ bond is prolonged by $0.04 \AA$ (from 1.13 to $1.17 \AA$ ).

It could be noted that the $\mathrm{O}-\mathrm{O}(\mathrm{C}-\mathrm{O})$ bond has elongated after $\mathrm{O}_{2}(\mathrm{CO})$ adsorption. As known, this elongation of molecule bond due to the foreign electrons entering into the $2 \pi *$ orbital and then the $2 \pi^{*}$ orbital has been broadened. ${ }^{14}$ From the energetic point of view, $E_{\text {ads }}\left(\mathrm{O}_{2}\right)$ is very closely with $E_{\text {ads }}(\mathrm{CO})$ (see Table 2) so that these two kinds of gas molecules have the same ability of competition on the anchored Ni atom. Furthermore, the coadsorption energy with the most stable coadsorption configuration of $\mathrm{CO}$ and $\mathrm{O}_{2}$ on the same anchored $\mathrm{Ni}$ atom is $-2.70 \mathrm{eV}$, which is lower than $E_{\text {ads }}(\mathrm{CO})$ or $E_{\text {ads }}\left(\mathrm{O}_{2}\right)$. In other words, the coadsorption is preferable than single gas molecule (CO or $\mathrm{O}_{2}$ ) adsorption. Thus, the preferable coadsorption configuration means that the Langmuir-Hinshelwood (LH) mechanism is expected to prevail over the Eley-Rideal (ER) mechanism for CO oxidation on Ni-3N-G.

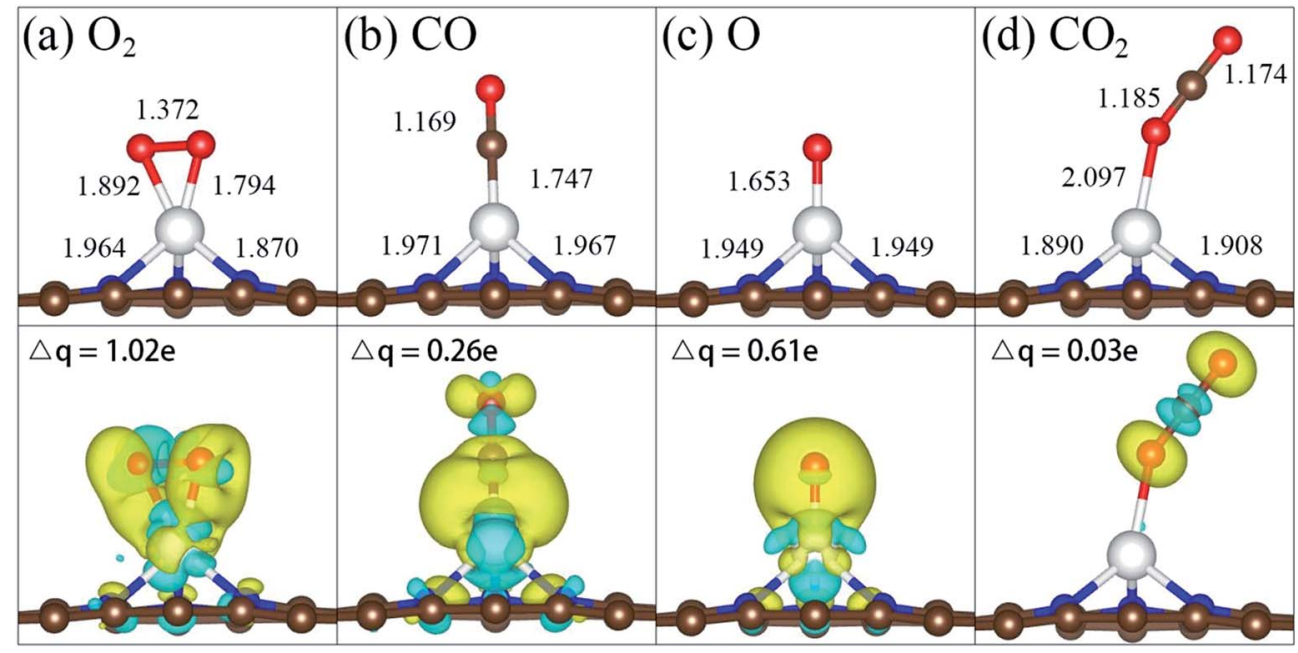

Fig. 3 The optimized geometric configurations (top panel) and isosurface plot of charge density difference (bottom panel) of $\mathrm{O}_{2}$ (a), $\mathrm{CO}$ (b), $\mathrm{O}$ (c) and $\mathrm{CO}_{2}$ (d) adsorbed on Ni-3N-G. The brown, blue, white and red spheres represent the $\mathrm{C}, \mathrm{N}, \mathrm{Ni}$ and $\mathrm{O}$ atoms, respectively. The faint yellow and azure areas represent charge accumulation and depletion, respectively. 
For single $\mathrm{O}$ atom adsorption, it has a lower adsorption energy $(-4.81 v s .-2.06 \mathrm{eV})$ and a shorter $\mathrm{O}-\mathrm{Ni}$ bond (1.653 vs. $1.794 \AA$ ) in comparison to $\mathrm{O}_{2}$. The adsorbed $\mathrm{O}$ atom gains $0.61 e$ charge from the Ni-3N-G, especially Ni. These results mean that single $\mathrm{O}$ atom could be stably adsorbed on $\mathrm{Ni}$.

As for $\mathrm{CO}_{2}$ adsorption, the most stable configuration with $E_{\text {ads }}\left(\mathrm{CO}_{2}\right)=-0.16 \mathrm{eV}$. The $E_{\text {ads }}\left(\mathrm{CO}_{2}\right)$ is very closely to that of Auembedded graphene $(-0.13 \mathrm{eV}) .{ }^{12}$ The corresponding charge density redistribution (as depicted in bottom panel of Fig. 3d) shows that charges are entirely accumulated at the two $\mathrm{O}$ atoms of $\mathrm{CO}_{2}$ molecule, which is nearly the same as that of a free $\mathrm{CO}_{2}$. The above results indicate a very weak interaction between $\mathrm{CO}_{2}$ and Ni-3N-G substrate. Therefore, the weak interaction will help $\mathrm{CO}_{2}$ desorption.

\subsection{CO oxidation reaction on Ni-3N-G}

As far as we know, for the reaction of CO oxidation, the Langmuir-Hinshelwood (LH) and the Eley-Rideal (ER) mechanisms are two well-known mechanisms. For the LH mechanism, considering the most stable energetically coadsorption configuration as initial state (LH-IS) and a stable peroxo-type OOCO intermediate as middle state (LH-MS). The ER mechanism means that $\mathrm{O}_{2}$ molecule will first take the active position and then reacts with nearby $\mathrm{CO}$ molecule. In order to figure out the favorable $\mathrm{CO}$ oxidation pathway on Ni-3N-G, both two mechanisms will be comparatively studied.

Before investigate the ER mechanism, we focus on the adsorption and dissociation of $\mathrm{O}_{2}$ on Ni-3N-G. When $\mathrm{O}_{2}$ reacts with $\mathrm{Ni}-3 \mathrm{~N}-\mathrm{G}$, we find that $\mathrm{O}_{2}$ can be adsorbed stably and is not easy to decompose energetically after $\mathrm{O}_{2}$ adsorption, which means that the breaking of $\mathrm{O}-\mathrm{O}$ bond may require more energy. For the ER reaction, the local configurations at each state along the reaction pathways are displayed in Fig. 4. The configuration of physisorbed $\mathrm{CO}$ nearby the preadsorbed $\mathrm{O}_{2}$ about $3 \AA$ A was chosen as the initial state (ER-IS). A transition state (ER-TS) is formed as the $\mathrm{CO}$ gradually approaches the activated $\mathrm{O}_{2}$ molecule, where $\mathrm{O}_{2}$ is apart from CO $1.584 \AA$ A. Finally, a $\mathrm{CO}_{2}$ molecule is generated and leaving an atomic $\mathrm{O}$ on $\mathrm{Ni}$. This pathway $(\mathrm{CO}+$ $\mathrm{O}_{2} \rightarrow \mathrm{CO}_{2}+\mathrm{O}$ ) needs at least $0.98 \mathrm{eV}$ to overcome such a high activation barrier, which by an energy release of $2.59 \mathrm{eV}$. The ER pathway of $\mathrm{CO}$ oxidation catalyzed by Ni-3N-G, without a carbonate-like intermediate state, is very similar to the ER pathway on the Fe-BN. ${ }^{32}$ Considering the high activation barrier of $\mathrm{O}_{2}$ molecule via the ER mechanism, we will focus on the $\mathrm{LH}$ mechanism in the following sections.

For the LH mechanism, the processes can be described with five stages in Fig. 5. The most stable coadsorption configuration was chosen as the initial state (LH-IS), where CO and $\mathrm{O}_{2}$ are both tilted to Ni-3N-G substrate. In the LH-IS, the distance of $\mathrm{C}-\mathrm{O}$ and $\mathrm{O}-\mathrm{O}$ bonds are prolonged by $0.02 \AA$ (from 1.14 to 1.16 $\AA$ ) and $0.03 \AA$ (from 1.26 to $1.29 \AA$ ), respectively.

Then, the top $\mathrm{O}$ atom in the $\mathrm{O}_{2}$ molecule comes near the $\mathrm{C}$ atom in $\mathrm{CO}$ to reach the transition state (LH-TS1). The barrier from LH-IS to LH-TS1 is estimated to be $0.61 \mathrm{eV}$. Third, the LHTS1 will come to the intermediate state (LH-MS) after relaxation. The distance of $\mathrm{O}-\mathrm{O}$ bond is prolonged by $0.232 \AA$ (from 1.290 to $1.522 \AA)$ in the processes from LH-IS to LH-MS. Finally, the reaction pathway proceeds from LH-MS to LH-FS with a relatively lower barrier of $0.51 \mathrm{eV}$. The $\mathrm{CO}_{2}$ molecule can be easily desorbed at room temperature due to the heat released during this process.

Furthermore, as shown in Fig. 6, we also checked the catalytic activity of the remaining atomic $\mathrm{O}$ on $\mathrm{Ni}$ atom. When a $\mathrm{CO}$ surround the $\mathrm{O}$ in $3 \AA$, the reaction will take place to produce

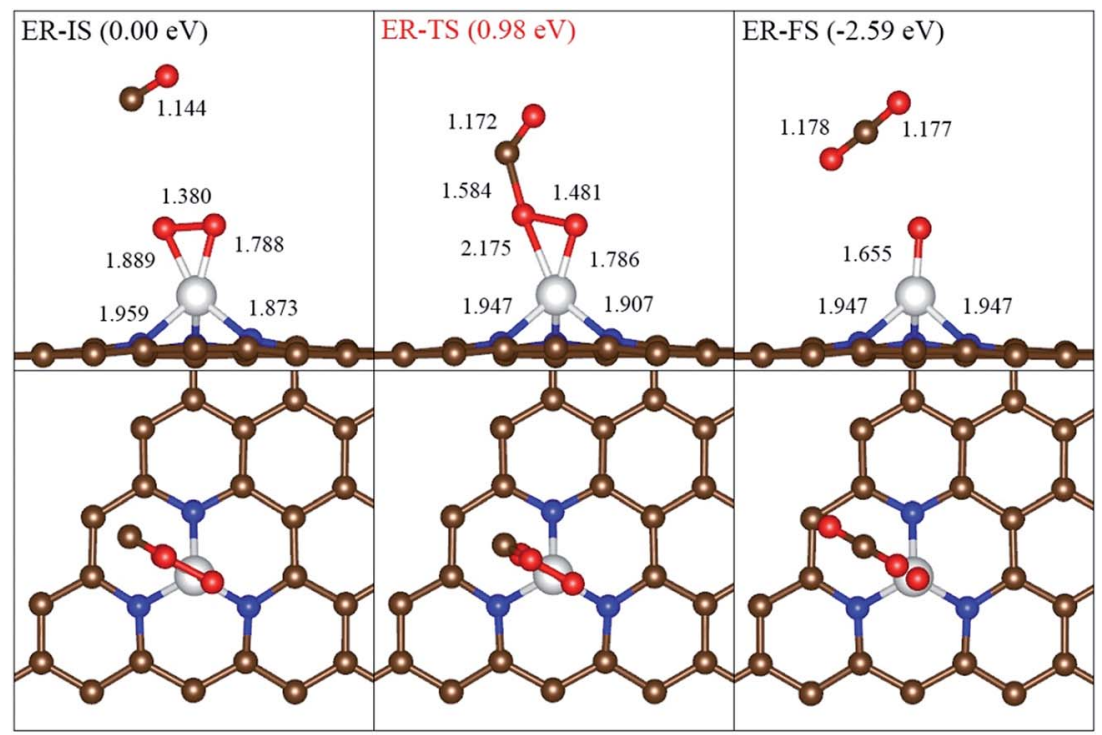

ER reaction: $\mathrm{CO}+\mathrm{O}_{2} \rightarrow \mathrm{CO}_{2}+\mathrm{O}$

Fig. 4 Top views (top panel) and side views (bottom panel) of local configurations of the initial state (ER-IS), transition state (ER-TS) and final state (ER-FS) for CO oxidation on Ni-3N-G via the ER mechanism. The brown, blue, white and red spheres represent the C, N, Ni and O atoms, respectively. 


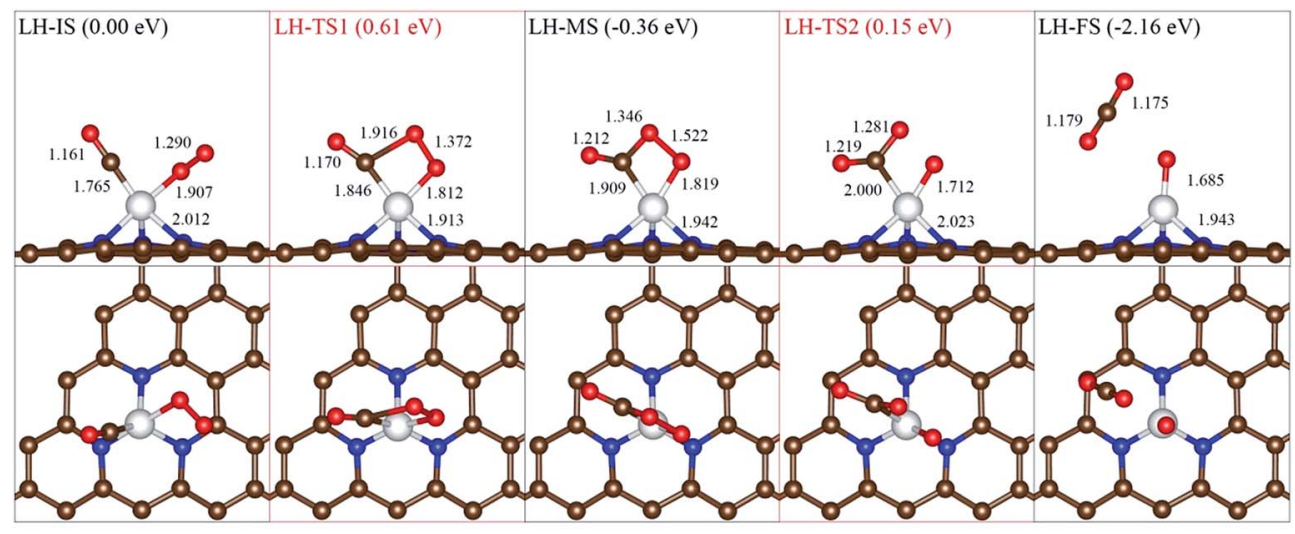

LH Reaction: $\mathrm{CO}+\mathrm{O}_{2} \rightarrow \mathrm{OOCO} \rightarrow \mathrm{CO}_{2}+\mathrm{O}$

Fig. 5 Top views (top panel) and side views (bottom panel) of local configurations of the initial state (LH-IS), transition state (LH-TS1 and LH-TS2), intermediate state (LH-MS) and final state (LH-FS) for CO oxidation on Ni-3N-G via the LH mechanism. The brown, blue, white and red spheres represent the $\mathrm{C}, \mathrm{N}, \mathrm{Ni}$ and $\mathrm{O}$ atoms, respectively.

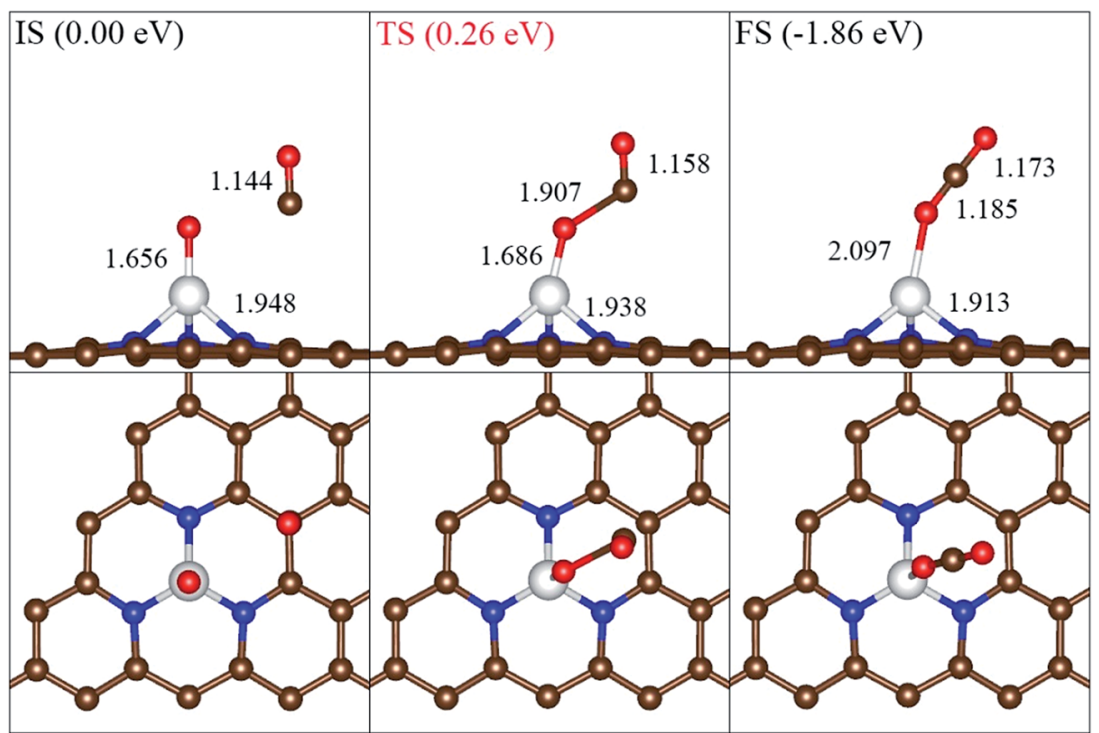

Fig. 6 The minimum energy path for $\mathrm{CO}$ oxidation with atomic $\mathrm{O}$. The brown, blue, white and red spheres represent the $\mathrm{C}, \mathrm{N}, \mathrm{Ni}$ and $\mathrm{O}$ atoms, respectively.

$\mathrm{CO}_{2}$. For this reaction progress, our result shows that the energy barrier is estimated to be $0.26 \mathrm{eV}$, which is much lower than that of the corresponding progress of Fe-embedded graphene. ${ }^{15}$ As mentioned above, the adsorption of $\mathrm{CO}_{2}$ on Ni-3N-G is very weak. Therefore, a large energy release of $1.86 \mathrm{eV}$ for this reaction step will facilitates $\mathrm{CO}_{2}$ desorption and regeneration of active site.

On the basis of above discussion, we conclude that the complete reactions for $\mathrm{CO}$ oxidation on Ni-3N-G may be described a two-step process: the $\mathrm{LH}$ reaction with an activation barrier of $0.61 \mathrm{eV}$ for OOCO formation as a starting step, followed by the process of $\mathrm{CO}$ oxidized by atomic $\mathrm{O}$ with a much lower barrier of $0.26 \mathrm{eV}$.

Compared to some previous results, the catalytic activity on Ni-3N-G for CO oxidation is slightly lower than that of some metal-graphene catalysts ( $\mathrm{Au} 0.31 \mathrm{eV}$; Pt $0.46 \mathrm{eV}$; Cu $0.54 \mathrm{eV}$; Fe
$0.58 \mathrm{eV}),{ }^{12-15}$ which is better than Fe-anchored graphene oxide $(0.7 \mathrm{eV}),{ }^{33} \mathrm{Pt}$ atom supported on oxidized graphene $(0.76 \mathrm{eV})^{34}$ or Co-3N-G $(0.86 \mathrm{eV}) .{ }^{25}$ In addition, Ni-3N-G has a similar activation barrier with Fe-embedded boron nitride monolayer $(0.61 \mathrm{eV}) .^{32}$ Then, the activation barrier of $0.61 \mathrm{eV}$ shows that Ni-3N-G has a high catalytic activity for CO oxidation.

\section{Conclusion}

In summary, we investigated the stability and catalytic activity of Ni-3N-G model catalyst by first-principles calculations. The Vsite could be considered as the most favorable adsorption site for single Ni atom adsorbed on the pyri-3N-G. The single $\mathrm{Ni}$ is hard to escape from the $\mathrm{V}$-site. The coadsorption of $\mathrm{CO}$ and $\mathrm{O}_{2}$ is preferable than single $\mathrm{CO}$ or $\mathrm{O}_{2}$ adsorption energetically. Furthermore, the activation barrier of $0.98 \mathrm{eV}$ for the ER 
mechanism is higher than that of $0.61 \mathrm{eV}$ for the $\mathrm{LH}$ mechanism with forming an intermediate OOCO, which indicates the $\mathrm{LH}$ mechanism is more favorable than the ER mechanism for CO oxidation. In general, a catalyst with an activation barrier of $0.61 \mathrm{eV}$ can be considered as one with a high activity for using at room temperature. Therefore, the Ni-3N-G is a promising candidate catalyst for $\mathrm{CO}$ oxidation, which has a potential advantage to develop inexpensive and efficient catalyst.

\section{Conflicts of interest}

There are no conflicts to declare.

\section{Acknowledgements}

This work was financially supported by the National Natural Science Foundation of China (No. 11575129 and 11275142) and the National Key Research and Development Program of China (No. 2017YFF0106506).

\section{References}

1 N. Lopez and J. K. Nørskov, J. Am. Chem. Soc., 2002, 124, 11262-11263.

2 R. Siburian, T. Kondo and J. Nakamura, J. Phys. Chem. C, 2013, 117, 3635-3645.

3 W. Yu, M. D. Porosoff and J. G. Chen, Chem. Rev., 2012, 112, 5780-5817.

4 R. Toyoshima, M. Yoshida, Y. Monya, Y. Kousa, K. Suzuki, H. Abe, B. S. Mun, K. Mase, K. Amemiya and H. Kondoh, J. Phys. Chem. C, 2012, 116, 18691-18697.

5 S. Shan, V. Petkov, L. Yang, J. Luo, P. Joseph, D. Mayzel, B. Prasa, L. Y. Wang, M. Engelhard and C.-J. Zhong, J. Am. Chem. Soc., 2014, 136, 7140-7151.

6 M. S. Chen, Y. Cai, Z. Yan, K. K. Gath, S. Axnanda and D. W. Goodman, Surf. Sci., 2007, 601, 5326-5331.

7 Z.-P. Liu and P. Hu, J. Chem. Phys., 2001, 115, 4977-4980.

8 Q. G. Jiang, Z. M. Ao, S. Li and Z. Wen, RSC Adv., 2014, 4, 20290-20296.

9 Y. Wang, Y. Y. Shao, D. W. Matson, J. H. Li and Y. H. Lin, ACS Nano, 2010, 4, 1790-1798.

10 Z. Q. Luo, S. H. Lim, Z. Q. Tian, J. Z. Shang, L. F. Lai, B. MacDonald, C. Fu, Z. X. Shen, T. Yu and J. Lin, J. Mater. Chem., 2011, 21, 8038-8044.

11 H. T. Liu, Y. Q. Liu and D. B. Zhu, J. Mater. Chem., 2011, 21, 3335-3345.
12 Y. H. Lu, M. Zhou, C. Zhang and Y.-P. Feng, J. Phys. Chem. C, 2009, 113, 20156-20160.

13 X. Liu, Y. H. Sui, T. Duan, C. G. Meng and Y. Han, Phys. Chem. Chem. Phys., 2014, 16, 23584-23593.

14 E. H. Song, Z. Wen and Q. Jiang, J. Phys. Chem. C, 2011, 115, 3678-3683.

15 Y. F. Li, Z. Zhou, G. T. Yu, W. Chen and Z. F. Chen, J. Phys. Chem. C, 2010, 114, 6250-6254.

16 M. Cueto, P. Ocón and J. M. L. Poyato, J. Phys. Chem. C, 2015, 119, 2004-2009.

17 X. W. Bai, E. J. Zhao, K. Li, Y. Wang, M. G. Jiao, F. He, X. X. Sun, H. Sun and Z. J. Wu, Carbon, 2016, 105, 214-223.

18 X. L. Zhang, Z. S. Lu, F. Zhang, Y. N. Tang and D. W. Ma, J. Power Sources, 2015, 276, 222-229.

19 Y. N. Tang, Z. Y. Liu, X. Q. Dai, Z. X. Yang, W. G. Chen, D. W. Ma and Z. S. Lu, Appl. Surf. Sci., 2014, 308, 402-407.

20 L. T. Qu, Y. Liu, J.-B. Baek and L. M. Dai, ACS Nano, 2010, 4, 1321-1326.

21 K. X. Niu, B. P. Yang, J. F. Cui, J. T. Jin, X. G. Fu, Q. P. Zhao and J. Y. Zhang, J. Power Sources, 2013, 243, 65-71.

22 L. Lin, Q. Zhu and A.-W. Xu, J. Am. Chem. Soc., 2014, 136, 11027-11033.

23 W. Orellana, J. Phys. Chem. C, 2013, 117, 9812-9818.

24 X. Liu, Y. H. Sui, T. Duan, C. G. Meng and Y. Han, Catal. Sci. Technol., 2015, 5, 1658-1667.

25 X. L. Zhang, Z. S. Lu and Z. X. Yang, J. Mol. Catal. A: Chem., 2016, 417, 28-35.

26 G. Henkelman, B. P. Uberuaga and H. Jónsson, J. Chem. Phys., 2000, 113, 9901-9904.

27 G. Henkelman and H. Jónsson, J. Chem. Phys., 2000, 113, 9978-9985.

28 T. Zhu, J. Li and S. Yip, Phys. Rev. Lett., 2004, 93, 025503.

29 G. Kresse and J. Furthmüller, Phys. Rev. B: Condens. Matter Mater. Phys., 1996, 54, 11169.

30 J. P. Perdew, K. Burke and M. Ernzerhof, Phys. Rev. Lett., 1996, 77, 3865.

31 G. Henkelman, A. Arnaldsson and H. Jónsson, Comput. Mater. Sci., 2006, 36, 354-360.

32 P. Zhao, Y. Su, Y. Zhang, S.-J. Li and G. Chen, Chem. Phys. Lett., 2011, 515, 159-162.

33 F. Y. Li, J. J. Zhao and Z. F. Chen, J. Phys. Chem. C, 2012, 116, 2507-2514.

34 Y. N. Tang, X. Q. Dai, Z. X. Yang, L. J. Pan, W. G. Chen, D. W. Ma and Z. S. Lu, Phys. Chem. Chem. Phys., 2014, 16, 7887-7895. 